\title{
MODELLING COAL COMBUSTION CONDITIONS AT ITS STORAGE IN A STACK
}

\author{
A.S. Masanik ${ }^{1}$, and $A$.V. Vasilevskiy ${ }^{1, *}$ \\ ${ }^{1}$ National Research Tomsk Polytechnic University, 634050 Tomsk, Russia
}

\begin{abstract}
A mathematical model describing the heat and mass transfer processes during storage and ignition of coal stored in the stack, has been proposed. Numerical studies have allowed to determine the composition of gases and temperature profiles in the depth of the stack, depending on the air temperature and cloud cover. It was found that the cloudless hot weather (daytime temperatures of $303 \mathrm{~K}$ ) within three days the top of the stack is heated to a temperature is its fire.
\end{abstract}

\section{Introduction}

Russian power industry is the largest consumer of primary fuel resources in the world. On a share of Thermal power stations account for about $30 \%$ of coal and more than $25 \%$ of natural gas produced in the country, and $10 \%$ of the produced oil. Thus the share of electricity in the internal coal consumption is $50 \%$, and natural gas - $40 \%$. These data indicate that the coal reserves are large in Russia.

It is known that coal can ignite spontaneously, especially when improper storage or in hot sunny weather. In many cases, the cause of fires in coal bunkers, and the open spaces is spontaneous combustion. Therefore, a central place in this area takes the problems of increasing the reliability of solid fuel storage. This problem can be solved by creating a scientific basis predictive modeling processes that occur during storage of solid fuels. Relevance topics of the research defined by its focus on creating the scientific foundations of security during storage and processing of natural solid fuels.

\section{Formulation of the problem}

Considered coal stack onto the upper surface of which can affect the convective and (or) the radiative heat flow. The aim is to determine the conditions of the surface layer stack heat and mass transfer with the environment (the conditions of the thermal effects on the stack), in which coal ignition in a stack may occur.

A mathematical model of heat and mass transfer, and coal ignition which is stored in the stack consists of a system of equations [1]:

mass conservation of the original condensed matter (coal)

*Corresponding author: subbot@tpu.ru 


$$
\rho_{1} \frac{\partial \varphi_{1}}{\partial t}=-R_{\mathrm{p}}, \quad R_{\mathrm{p}}=k_{\mathrm{p}} \rho_{1} \varphi_{1} \exp \left(-\frac{E_{\mathrm{p}}}{R T}\right)
$$

mass conservation of water in a bound state

$$
\rho_{2} \frac{\partial \varphi_{2}}{\partial t}=-R_{\mathrm{L}}, \quad R_{\mathrm{L}}=k_{\mathrm{L}} \rho_{2} \varphi_{2} \exp \left(-\frac{E_{\mathrm{L}}}{R T}\right)
$$

mass conservation of coke

$$
\rho_{3} \frac{\partial \varphi_{3}}{\partial t}=\alpha_{\mathrm{C}} R_{\mathrm{P}}-s R_{\mathrm{C}}, \quad R_{\mathrm{C}}=\frac{M_{\mathrm{C}}}{M_{\mathrm{O}_{2}}} k_{\mathrm{C}} \rho_{5} \varphi_{5} \varphi_{3} \exp \left(-\frac{E_{\mathrm{C}}}{R T}\right)
$$

mass conservation of ash

$$
\rho_{3} \frac{\partial \varphi_{3}}{\partial t}=\alpha_{S} R_{\mathrm{P}}, \text { where } \alpha_{\mathrm{C}}=\frac{v_{3}^{\prime} M_{3}}{v_{1} M_{1}}, \quad \alpha_{\mathrm{S}}=\frac{v_{4}^{\prime} M_{4}}{v_{1} M_{1}}, \quad \sum_{\mathrm{j}=1}^{5} \varphi_{\mathrm{j}}=1 ;
$$

the continuity of the gas phase

$$
\frac{\partial}{\partial t}\left(\rho_{5} \varphi_{5}\right)+\frac{\partial}{\partial z}\left(\rho_{5} \varphi_{5} v\right)=\alpha_{\mathrm{G}} R_{\mathrm{P}}+R_{\mathrm{L}}+s R_{\mathrm{C}}, \quad \alpha_{\mathrm{G}}=\sum_{\alpha=2}^{5} \frac{v_{5 \alpha}^{\prime} M_{5 \alpha}}{v_{1} M_{1}}
$$

mass conservation $\alpha$-gas phase constituents

$$
\frac{\partial}{\partial t}\left(\rho_{5} \varphi_{5} c_{\alpha}\right)+\frac{\partial}{\partial z}\left(\rho_{5} \varphi_{5} v c_{\alpha}\right)=\frac{\partial}{\partial z}\left(\rho_{5} \varphi_{5} D_{\alpha} \frac{\partial c_{\alpha}}{\partial z}\right)+R_{5 \alpha}+R_{5 \mathrm{~S} \alpha}, \quad \alpha=1, \ldots, 6
$$

conservation of energy

$$
\sum_{j=1}^{5} \rho_{\mathrm{j}} \varphi_{\mathrm{j}} c_{\mathrm{pj}} \frac{\partial T}{\partial t}+\rho_{5} \varphi_{5} c_{\mathrm{p} 5} v \frac{\partial T}{\partial z}=\frac{\partial}{\partial z}\left(\lambda \frac{\partial T}{\partial z}\right)+q_{\mathrm{P}} R_{\mathrm{P}}-q_{\mathrm{L}} R_{\mathrm{L}}+q_{\mathrm{C}} s R_{\mathrm{C}}
$$

gas movement in the pores and state equation

$$
v=-\frac{k}{\mu}\left(\frac{\partial p}{\partial z}-\rho_{5} g\right), \quad p=\frac{\rho_{5} R T}{M}, \quad M=1 / \sum_{\alpha=1}^{7} \frac{c_{\alpha}}{M_{5 \alpha}} .
$$

The system of differential equations (1) - (8) was solved with the boundary conditions

$$
\begin{gathered}
t=0: \quad \varphi_{\mathrm{j}}=\varphi_{\mathrm{jH}}, \mathrm{j}=\overline{1,4}, \quad c_{\alpha}=c_{\alpha \mathrm{H}}, \alpha=\overline{1,6}, \quad p_{5}=p_{\mathrm{H}}, T=T_{\mathrm{H}} ; \\
z=0: \quad p=p_{\mathrm{e}}, \quad c_{\alpha}=c_{\alpha \mathrm{e}}, \quad \lambda \frac{\partial T}{\partial z}=\alpha_{1}\left(T-T_{\mathrm{e}}\right)+q_{\mathrm{R}} ; \\
z=\mathrm{H}: \quad p=p_{\mathrm{H}}, \quad \frac{\partial c_{\alpha}}{\partial z}=0, \quad \lambda \frac{\partial T}{\partial z}=\alpha_{2}\left(T-T_{\mathrm{w}}\right) .
\end{gathered}
$$


Here $v$ - filtration rate; $\alpha_{\mathrm{G}}, \alpha_{\mathrm{C}}, \alpha_{\mathrm{S}}$ - proportion of gaseous products, coke and ash formed during the pyrolysis of coal; $M$ - molecular weight gaseous products; quantities characterizing the condensed phase, attributed to index $s ; k$ - coal seam permeability coefficient; $T$ - the temperature in the coal stack; $E_{\mathrm{p}}, k_{\mathrm{p}}, E_{\mathrm{L}}, k_{\mathrm{L}}, E_{\mathrm{C}}, k_{\mathrm{C}}$ - activation energy and pre-exponential factor of coal pyrolysis reaction, evaporation of water and the heterogeneous reaction of coke oxidation, respectively; $R$ - universal gas constant; $D_{\alpha}, \lambda_{5 \alpha}, c_{\alpha}, M_{5 \alpha}-$ coefficients of molecular diffusion, thermal conductivity, mass concentration and the molecular weight of $\alpha$ components , parameter with index $\alpha=1$ corresponds to the component $\mathrm{O}_{2}, \alpha=2-\mathrm{CO}, \alpha=3-$ $\mathrm{CO}_{2}, \alpha=4-\mathrm{H}_{2}, \alpha=5-\mathrm{CH}_{4}, \alpha=6-\mathrm{H}_{2} \mathrm{O}, \alpha=7-\mathrm{N}_{2} ; p$ - pressure gaseous products in the pores; $p_{\mathrm{H}}-$ external atmospheric pressure; $s$ - pore specific surface; $q_{\mathrm{R}}$ - radiative flux from the external environment on the surface of the coal; $t$ - time; $z$-spatial variable, directed from the top surface stack vertically downwards; $q_{\mathrm{R}}$ и $T_{\mathrm{E}}$ - function of time (depending on time of day), $\alpha_{1}$ и $\alpha_{2}$ - heat transfer coefficients characterizing the convective heat transfer on the top and bottom surfaces of stack; $\rho_{\mathrm{i}}, c_{\mathrm{pi}}, \lambda_{\mathrm{i}}, \varphi_{\mathrm{i}}-$ true density, heat capacity, thermal conductivity and the volume fraction of coal $-\mathrm{i}=1$, water $-\mathrm{i}=2$, coke $-\mathrm{i}=3$, ash $-\mathrm{i}=4$ and gas phase $-\mathrm{i}=5 ; \lambda_{\mathrm{S}}, \lambda_{5}, \lambda_{\mathrm{R}}-$ coefficient of thermal conductivity of the condensed phase (coal) and the coefficients of molecular and radiant thermal conductivity the gaseous mixture; $\sigma-$ Stefan-Boltzmann constant; $\mu$ - dynamic viscosity factor; $\beta$ - constant in the quadratic Darcy law; $g$ - acceleration of gravity; $v$ - filtration rate.

\section{The method of solving and analysis of numerical results}

The boundary value problem (1) - (11) was solved numerically, use iterative-interpolation method [2]. Based on the analysis of the results, it was found that in dry hot weather, coal stack will not light up if it does not get sunlight, that is when only the convective heat transfer is carried out. There were also calculations when on the surface of the coal stacks impacted convective and radiative heat flow. Before surface of the earth in western Siberia in cloudless weather, depending on the degree of atmospheric dustiness, reaches the maximum radiative heat flow equal to $800 \div 1200 \mathrm{~W} / \mathrm{m}^{2}$. Therefore, for the heat equation we considered the boundary conditions that take into account the air temperature at night dropped to $293 \mathrm{~K}$, and in the daytime rose to $303 \mathrm{~K}$. Radiative flux was zero at night, and during the day rose to $800 \div 1200 \mathrm{~W} / \mathrm{m}^{2}$.

Figure 1 shows a heating of coal at the upper surface of the stack, when the radiation stream by day increased to $1000 \mathrm{~W} / \mathrm{m}^{2}$. As can be seen from the graph, in such effect heat flow to stack have happened fire in the coal layer depth a distance of approx $0.05 \mathrm{~m}$ from the surface. 


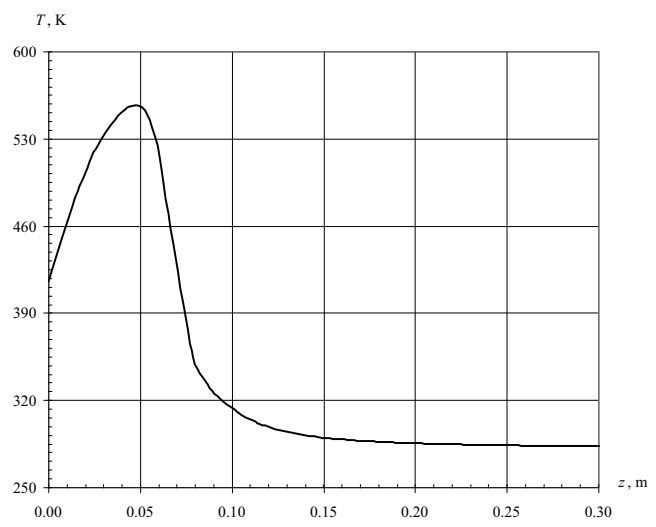

Fig. 1. Distribution of temperature at the upper surface of the stacks.

Figure 2 shows the temperature change with time at interior points the coal stacks: the upper curve 1 at a distance of $0.005 \mathrm{~m}$ from the surface, average $2-0.05 \mathrm{~m}$ and lower $3-$ $0.1 \mathrm{~m}$.

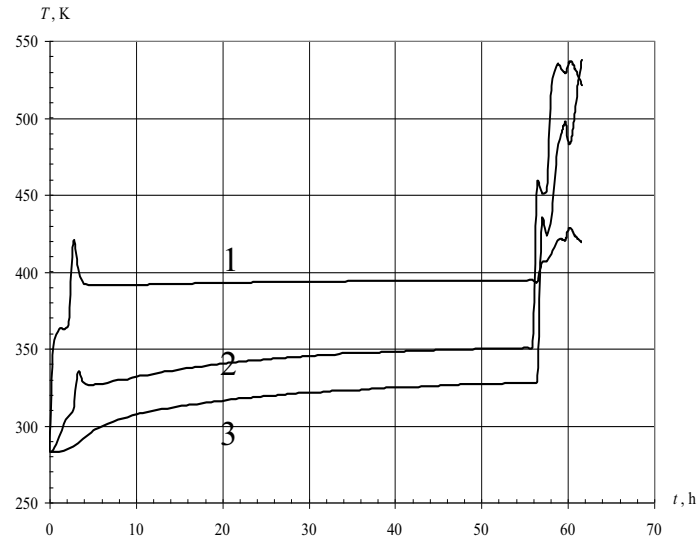

Fig. 2. The temperature change over time in some points of the coal seam.

Analyzing Figure 2 it can be stated, that at simultaneous effects of on the coal stack convective and radiative flux on the third day there was an ignition of coal stored in the stack.

\section{Conclusions}

Thus, we can conclude the following: during storage of coal in the stack are not protected from the sun in hot cloudless weather may cause it to catch fire. In the considered case, ignition has occurred in about three days.

\section{References}

1. R.N. Kulesh, A.N. Subbotin, Nat. Eng. Sc. 4 (2014)

2. A. M. Grishin, V. I. Zinchenko, K. N. Efimov, A. N. Subbotin, A. S. Yakimov. Iterationinterpolation method and its application (Publ. house TGU, Tomsk, 2004) 